\title{
Über das Wesen der psychotischen Halluzinationen: Eine lacansche Sichtweise ${ }^{1}$
}

\author{
Stijn Vanheule (Gent)
}

\begin{abstract}
Zusammenfassung: Dieser Aufsatz gibt eine Übersicht über die von Lacan in den 1950er-Jahren entwickelte Theorie der Halluzinationen. Lacan bricht mit der traditionellen Sichtweise, welche von der Unwirklichkeit der halluzinatorischen Wahrnehmung ausgeht und untersucht stattdessen die Auswirkungen von psychotischen Halluzinationen auf die Person, welche sie erlebt. Er entwickelt ein alternatives theoretisches Bezugssystem, aus welchem hervorgeht, dass die Psychose durch die Unfähigkeit gekennzeichnet ist, die eigene Existenz als Subjekt in Beziehung zum Andern zu signifizieren. Lacans Theorie wird sowohl auf der konzeptuellen wie auch auf der klinischen Ebene erörtert, zum einen anhand von Material aus einer seiner klinischen Falldarstellungen und zum anderen anhand von Ausschnitten seiner Diskussion des Falles Schreber.
\end{abstract}

Schlüsselwörter: Lacan, Halluzination, Psychose, Psychoanalyse, Wahrnehmung

\section{Einführung}

Im vorliegenden Aufsatz befasse ich mich mit Lacans Theorie der psychotischen Halluzinationen, die er in den 1950er-Jahren entwickelt hat. Seine Theorie ist insofern innovativ, als sie eine Anzahl von allgemein anerkannten Annahmen in der Erforschung der Halluzinationen untergräbt. Lacan sagt sich von den gebräuchlichen Definitionen der Halluzinationen los, in welchen der Fokus auf der Unwirklichkeit der halluzinatorischen Wahrnehmungen liegt, und rückt stattdessen deren Wirkung auf die halluzinierende Person ins Zentrum seiner Betrachtungen. Im Laufe seines Werks entwickelt Lacan zudem immer wieder neue theoretische Konzepte, welche es ihm ermöglichen, sich dem Phänomen der Halluzinationen aus einer Perspektive zu nähern, die bis anhin noch nie eingenommen worden war. Er distanziert sich von einer Phänomenologie und Psychopathologie der Wahrnehmung, um zu einer Theorie der Logik des Subjektes zu gelangen. Damit stellt uns Lacan eine neue Sprache zur Verfügung, welche ein anderes Modell für die Reflexion auf die Psychose erlaubt. Er gelangt zu einem grundlegend neuen Ansatz für die Klinik der Psychose, der über das Konzept der Halluzination als 
Zeichen eines pathologischen Prozesses hinausgeht und die subjektive Wirkung der halluzinatorischen Erfahrung ergründet.

Das Verdienst seiner Theorie ist zweifach. Einerseits skizziert Lacan ein neues Bezugssystem, welches mit vielen Annahmen der Psychiatrie bricht und sieht stattdessen die Psychose als Kennzeichen eines Unvermögens, die eigene Existenz als Subjekt in Beziehung zum Anderen zu signifizieren. Die Konfrontation mit der Andersheit des Anderen eröffnet ein Gebiet der Unsinnigkeit, in welchem das Identitätsgefühl suspendiert wird. Halluzinationen erlangen einen Status, der mit der psychotischen Erfahrung der Unsinnigkeit und des Aufschubs korreliert und der von der Verkörperung des auslösenden Moments bis zum Versuch reicht, die Erfahrung der Destabilisierung zu mildern. In Lacans Erörterungen existiert kein eindeutiges nomothetisches Modell der subjektiven Wirkung der Halluzinationen; ihr Status muss von Fall zu Fall neu untersucht werden. Andererseits geht es hier um eine pragmatische und ethische Frage: Lacans ideographischer Ansatz ist eine Aufforderung an die Kliniker zu «einer totalen, wenn auch bewussten Unterwerfung unter die eigentlichen subjektiven Positionen des Kranken» (1959/1986, S. 66). Mit anderen Worten: Der Kliniker soll - ausgehend von einer klaren Vorstellung über das Wesen der Halluzinationen - in einen Dialog mit dem halluzinierenden Patienten eintreten; in einen Dialog, der sich ausschliesslich nach der singulären Erfahrung und der Rede der halluzinierenden Person richtet. Auf der klinischen Ebene wird durch Lacans Theorie ein neuer Approach zur Psychose initiiert, welcher der Anwendung von standardisierten Behandlungsprotokollen zuwiderläuft und stattdessen den Fokus auf die ideographische Ermittlung des jeweiligen Status' der Halluzinationen des Patienten legt.

Im Zentrum meiner Diskussion stehen Lacans Text «Über eine Frage, die jeder möglichen Behandlung der Psychose vorausgeht» (1959/1986, S. 61-117), sein drittes Seminar von 1955-1956 sowie eine einjährige Untersuchung der Psychose (1981/1997). Ich setze mich mit den Konzepten beider Texte auseinander und stelle sie in den Kontext seiner späteren Theorie der Logik des Signifizierens. Auf Lacans weitere Ausarbeitung dieser Theorie werde ich im vorliegenden Artikel nicht eingehen. Lacan hat in den 1960er- und 70er Jahren bedeutende Änderungen an seinen Reflexionen vorgenommen und seine Konzepte des so genannten «object $a$ », der «jouissance» und des «sinthome» eingeführt. Mein vor Kurzem erschienenes Buch (Vanheule 2011) ist eine Erörterung der daraus resultierenden Änderungen in seiner Theorie der Psychose im Allgemeinen und seiner Theorie der Halluzinationen im Besonderen. 
Erstmalig in der Geschichte der Medizin definierte der französische Psychiater Jean-Etienne Esquirol Halluzinationen und zwar folgendermassen: «Ein Mensch, der die feste Überzeugung einer wirklich empfangenen Empfindung hat und zwar dann, wenn kein äusserer Gegenstand sich in der Umgebung seiner Sinne befindet, der diese Empfindung erregen könnte, ein solcher hat Hallucinationen» (Esquirol 1838, S. 95). Obgleich diese Definition zu einigen Debatten Anlass gab (Berrios 1996), waren sich viele Autoren in dem Punkt einig, dass eine Halluzination eine Wahrnehmungserfahrung sei, bei welcher ein entscheidender externer Stimulus fehle. So bezeichnete beispielsweise Lacans Freund und intellektueller Kontrahent Henry Ey die Halluzinationen als «Wahrnehmungen ohne wahrzunehmendes Objekt» (Ey 1973, S. 50). Neuere Studien beschreiben Halluzinationen als «jede Wahrnehmungserfahrung ohne externe Stimuli» (Allen, Laroi, McGuire \& Aleman 2008, S. 176), oder als «eine sinnliche Erfahrung, welche unter Abwesenheit einer entsprechenden externen Stimulation des relevanten Sinnesorganes auftritt und so real wirkt, dass sie einer echten Wahrnehmung ähnelt, worüber das Subjekt keine unmittelbare oder willentliche Kontrolle zu haben glaubt, und welche im Wachzustand auftritt» (David 2004, S. 110). Wie zu erkennen ist, liegt der Fokus all dieser Definitionen auf dem Erleben einer sinnlichen Wahrnehmung und betont die Unwirklichkeit der halluzinatorischen Wahrnehmung. Trotz teils unterschiedlichen Formulierungen ist die Ähnlichkeit mit Esqirols ursprünglicher Definition augenfällig.

\section{Eine Halluzination ist (k)ein objektloses Perceptum}

Lacan greift bei seiner Entwicklung einer neuen Theorie der Halluzinationen auf die vom Philosophen Merleau-Ponty entwickelten Konzepte zurück. 1945 erschien Merleau-Pontys Buch über die Phänomenologie der Wahrnehmung, welches Lacan offensichtlich entscheidend inspiriert hat, obwohl er es kaum zitiert (Kusnierek 2008; Merleau-Ponty 1945/1962; Miller 2001). In seinem Buch hält Merleau-Ponty fest, dass es sich bei der menschlichen Wahrnehmung nicht nur um eine Frage der aufgrund sinnlicher Stimulation wahrgenommener Bilder handelt. Unser geistiges Bild der Welt (das «perceptum», «was gesehen wird») kann nicht nur in messbaren Fakten verstanden werden, weil das wahrnehmende Subjekt selbst (das «percipiens», «der, welcher sieht») immer Teil der von ihm wahrgenommenen Welt ist. Percipiens und perceptum sind eng miteinander verknüpft, weil der menschliche Körper massgeblich an der Wahrnehmung beteiligt ist. Laut Merleau-Ponty «versteht» unser Körper die Wahrnehmung und verleiht 
ihr Gestalt - er dient demnach nicht ausschliesslich der objektiven Verarbeitung sensorischer Information.

Merleau-Pontys Werk (1945/1962) ist wesentlich vom Konzept der Gestaltpsychologie geprägt; diese postuliert das Wahrnehmungsfeld an sich als organisiert (Miller 2001). Das menschliche perceptum verfügt über eine innere Logik oder Organisation, die sich nicht nur aus der externen Welt ableiten lässt. In seiner Kritik der gängigen psychiatrischen Ansätze beruft sich Lacan (1959/1986) auf diese Konzepte und weist darauf hin, dass die psychiatrischen Theorien im Wesentlichen auf der Vorstellung gründen, dass unser Sensorium Informationen von Objekten aus einer extramentalen materiellen Welt auswähle und das percipiens aufgrund dieser Informationen ein künstliches perceptum bilde. Lacan hält eine solche paradigmatische Vorstellung für typisch scholastisch und in ihrem Kern der Lehre von Thomas Aquinas über Wahrnehmung und Kognition verhaftet (Stump 2003). Wir können sie auch als linear bezeichnen. Sie geht von der Annahme aus, dass das percipiens dazu angeregt wird, ein perceptum zu bilden, wenn die Sinne durch ein extramentales Objekt stimuliert werden (siehe Abbildung 1).

\section{Figure $1 \quad$ Schematische Darstellung des klassischen Wahrnehmungsparadigmas}

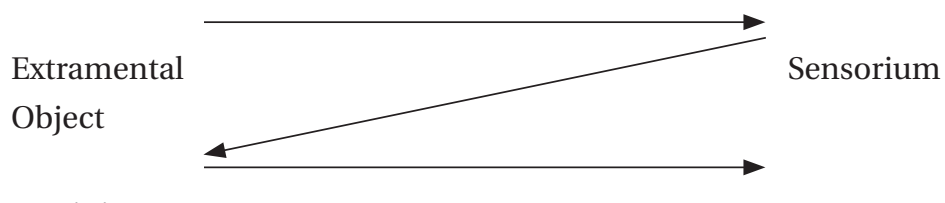

Percipiens

Perceptum

Zwar unterscheiden sich die psychiatrischen Definitionen der Halluzination stark voneinander, aber Lacan weist darauf hin, dass sich alle innerhalb des Rahmens der klassischen Wahrnehmungsparadigmen bewegen und damit auch das grundlegende Konzept der Halluzination als «objektloses perceptum» verkörpern (Lacan 1959/1986, S. 64). Obwohl kein extramentales Objekt existiert, welches eine sensorische Verarbeitung stimuliert, wird ein mentales Bild geschaffen. Er gibt zu bedenken, dass ein solches Konzept spezifische Fragen mit sich bringt. Einerseits werden dabei Halluzinationen entsprechend dem betreffenden Wahrnehmungsregister klassifiziert (visuelle Halluzinationen, auditive Halluzinationen, taktile Halluzinationen), andererseits führt dieses Konzept zu einer aussergewöhnlich beharrlichen Suche nach dem pathogenen Prozess im «percipiens», welcher für die Produktion von falschen «percepta» verantwortlich 
ist; einer Suche, welche noch immer für die empiristische Betrachtungsweise der zeitgenössischen psychiatrischen Forschung bestimmend ist.

Lacan betrachtet die erste Frage als irreführend. Er hält die sensorische Eigenschaft der Halluzinationen für vernachlässigbar und verweist auf das Beispiel der Taubstummen. Personen, welche von Geburt an stark schwerhörig sind und per definitionem nie auditive sensorische Information verarbeitet haben, können dennoch unter verbalen Halluzinationen leiden (siehe auch Atkinson 2006). Wie aber lässt sich dies erklären, wenn das auditive Register, welches bei Taubheit inaktiv ist, für akustische Halluzinationen unentbehrlich ist? Lacan folgert, der Akt des Hörens könne nicht in Bezug auf die involvierten sensorischen Register verstanden werden, sondern in Bezug auf bedeutungsstiftende Prozesse.

Die zweite Frage ist auch problematisch, weil sie impliziert, das «percipiens» sei eine Instanz, welche eingehende sensorische Information synthetisiert und aktiv Wahrnehmungen erzeugt. Lacan zufolge wirkt das «percipiens» nur dann einheitsstiftend, wenn die Person auf das Imaginäre zurückzugreifen vermag, mit anderen Worten: Das «percipiens» bildet nur dann sein eigenes «perceptum», wenn es «Gestalten»-oder konsistente mentale Repräsentationen und Bilder produzieren kann. Das Imaginäre ermöglicht einer Person, in sich geschlossene und kohärente Erfahrungen zu erzeugen und einen Realitätssinn zu entwickeln (Lacan 1949/1975). Aber diese vereinheitlichende Tendenz widerspiegelt nur eine (Hervorhebung der Übers.) Dimension unseres jeweiligen Verhältnisses zur Welt. Lacan unterscheidet noch zwei weitere Dimensionen: «das Symbolische» und das «Reale». Das Symbolische bezeichnet die strukturimmanente Art, die Welt zu erfahren. Das Reale bezieht sich auf alle Dinge, die wir nicht begreifen können (Fink 1996, 1997; Nobus 2000). Lacan unterstreicht in seinem Konzept der verbalen Halluzinationen die Notwendigkeit, das Symbolische und das Reale zu berücksichtigen.

Ein aufgeschlossener Kliniker, so argumentiert er, könne gewöhnlich wenn er von einer begrifflichen Ebene ausgeht - erkennen, dass Patienten ihre Halluzinationen als nicht konsistent mit anderen Aspekten ihres mentalen Lebens erleben. Dies führt zu Zweifeln an einem Prinzip, welches auf der synthetisierenden Aktivität des Patienten selbst gründet. Im Gegenteil: Die Halluzination stellt den Patienten vor ein Problem und er empfindet sie als paradox. Lacan zufolge wird ein Kliniker, der aufmerksam hinhört und sich nicht von den theoretischen Annahmen eines pathologischen Prozesses im Patienten blenden lässt, gewöhnlich eine Anzahl von "Paradoxien [beobachten, welche das halluzinierende Subjekt] bei dieser eigenartigen Wahrnehmung erleidet»(1966/1986a, S. 65). Damit wird die Frage obsolet, wie ein halluzinierendes «percipiens» ein falsches «perceptum» 
schaffen könne: Lacan interessiert sich allein für die Art und Weise, wie das halluzinierte «perceptum» das «percipiens» affiziert. Ihm geht es einzig darum, dass eine halluzinierende Person nicht mit ihren Halluzinationen in Einklang steht, sondern ihnen unterworfen ist. Die Halluzination lässt das angeblich vereinheitlichende percipiens als äquivok erscheinen (ebenda).

Wir können diese Überlegungen mit Lacans Neurosentheorie in Verbindung bringen, in welcher das Subjekt «barriert» ( barré ) resp. «gespalten» ist, weil es nicht mit den Erzeugnissen seines Unbewussten zusammenfällt und davon ausgehen, dass für das psychotische Subjekt dasselbe gilt. In dem Masse, wie eine halluzinierende Person nicht mit ihren eigenen Halluzinationen zusammenfällt, ist auch sie ein gespaltenes Subjekt. Tatsächlich impliziert der Begriff des Subjekts in Lacans Theorie, dass ein externes Element (ein Signifikant) die eigene Identität determiniert (Fink 1996). Sowohl in der Neurose als auch in der Psychose ist das Subjekt eine determinierte Entität, und keine determinierende Kraft. Aus diesem Grunde wird ein lacanscher Psychoanalytiker in der klinischen Praxis sein Augenmerk auf die Konstellation der Signifikanten richten und damit dem Patienten die Möglichkeit bieten, diese Konstellation neu zu konfigurieren.

Folglich ist eine Halluzination kein objektloses «perceptum» sondern ein «perceptum», welches auf das «percipiens» eine paradoxe Wirkung ausübt. Wie diese paradoxe Wirkung verstanden werden kann, werde ich im nächsten Teil zu ergründen suchen.

\section{Eine Halluzination ist ein aus der Kette gefallener Signifikant - Teil I}

Um die paradoxeWirkung der Halluzinationen zu erforschen und die Struktur der halluzinatorischen Erfahrung im Allgemeinen zu entschlüsseln, berichtet Lacan (1981/1997, 1966/1996) von einer jungen Frau, die er 1955 anlässlich einer seiner klinischen Falldarstellungen befragt hat. In einer klinischen Falldarstellung - die sich an ein Publikum von Psychiatern und Psychoanalytikern wendet - schildert der mit dem jeweiligen Fall betreute Arzt die Geschichte eines/r seiner Patienten/ seiner Patientinnen und versucht, die Struktur der jeweiligen Probleme zu entschlüsseln (Miller 1980).

Hier handelt es sich um eine junge Frau, die zusammen mit ihrer Mutter, mit welcher sie sich in einem « délire à deux» befand, hospitalisiert wurde, weil sie von der wahnhaften Gewissheit besessen waren, belästigt und bedroht zu werden. Während seiner Fallvorstellung bezieht sich Lacan auf diese Gewissheit und bittet die Patientin, ihr Problem im Detail zu erklären. Die Patientin klagt unter anderem, dass sie und ihre Mutter von ihren Nachbarn beleidigt worden seien, eine Klage, 
auf die Lacan besonders eingehen wird. Anfänglich war ihnen der Kontakt mit den Nachbarn durchaus angenehm. Die Frau wurde zur Freundin; sie besuchte die Patientin und ihre Mutter häufig in deren Wohnung und man verstand sich gut. Von einem bestimmten Zeitpunkt an erlebten jedoch die Patientin und ihre Mutter die Besuche als eindringend. "Sie kam immer klopfen, wenn sie bei ihrer Toilette waren, oder im Augenblick, wo (...) sie gerade dabei waren, abendzuessen oder zu lesen» (Lacan 1981/1997, S. 61) und sie begannen, sich von der Nachbarin zu distanzieren. Zwar will die Patientin ihre Gewissheit nur widerwillig mit Beispielen dokumentieren, erzählt aber Lacan schliesslich folgende Anekdote: Eines Tages ging sie über den Flur ihres Mietshauses und wurde vom Liebhaber der Nachbarin beleidigt. Dieser Liebhaber hatte mit der Nachbarin eine Affäre, obwohl er verheiratet war, und wir wissen, dass ihn unsere Patientin als leichtlebig betrachtete (S. 59). Als sie ihm im Flur begegnete, halluzinierte sie das beleidigende Wort «Sau». Das Wort Sau ist für die Patientin eine Illustration dessen, was sie und ihre Mutter erlitten hatten; Lacan stellt aber fest, dass sie diesen bedeutungsschwangeren Begriff nur widerwillig über die Lippen bringt. Die junge Frau war nicht nur entsetzt:Was sie gehört hatte, verwirrte und verblüffte sie auch. Sie wusste nicht genau, was sie davon halten sollte und hatte deshalb Mühe, darüber zu sprechen. Und genau auf eine solche Erfahrung bezieht sich Lacan in seiner Feststellung, dass die Halluzination eine paradoxe Wirkung auf das «percipiens» habe; sie ist ein «perceptum», welche vom Patienten nicht mit anderen Erfahrungen in Einklang gebracht werden kann.

Ausserdem ist für Lacan diese Konfrontation mit einem Paradoxon in erster Linie ein Hinweis auf einen radikalen Einbruch im mentalen Leben. Lacan bezeichnet Halluzinationen als «Einbruch des Realen» (1959/1986, S. 68). Damit will er darauf hinweisen, dass eine Halluzination für den Patienten gewissermassen aus heiterem Himmel kommt, sie ist eine Begegnung mit einem unvorstellbaren Element, das sich von ausserhalb des mentalen Lebens aufdrängt. Eine Halluzination ist insofern «real», als sie ein «plötzliches Auftauchen einer totalen Fremdheit ist» (1981/1997, S. 104). Lacans Formulierung dieses Konzepts erinnert an die Auffassung des französischen Psychiaters Guiraud (1950), der psychotische Halluzinationen als «xenopathisch» bezeichnete, oder anders gesagt: Sie werden normalerweise als etwas erlebt, das von aussen kommt.

Der zweite Aspekt, den er hervorhebt, schreibt dem halluzinierten «perceptum» selbst eine interne Organisation zu und widerspiegelt damit das Konzept von Merleau-Ponty. Lacans Formulierung ist aber prägnanter: Er nimmt an, dass im Erleben einer Halluzination eine signifizierende Struktur erkannt wer-

Psychosen - Psychoanalytische Perspektiven 
den kann (1966/1986). Sein Konzept der signifizierenden Struktur von verbalen Halluzinationen bedeutet eine entscheidende Abweichung von Merleau-Pontys Philosophie (Miller, 2001). Um dies verständlich zu machen, werde ich kurz Lacans Theorie der Logik des Signifizierens erläutern und untersuchen, wie Bedeutung und Subjektivität durch den Gebrauch von Signifikanten generiert werden (Lacan, 1966/1986). Lacan hat diesen Bereich seiner Theorie parallel zu seiner Arbeit über Psychosen ausgearbeitet, aber die Grundbausteine finden sich auch in seinem Seminar (Lacan 1981/1993), vor allem in seinem Artikel über die Psychose (Lacan 1981/1997).

\section{Zwischenspiel: Die Logik des Signifizierens}

Lacan zufolge bestimmen zwei miteinander verbundene Dimensionen (in Abbildung 2 durch die zwei Pfeile dargestellt) unseren Gebrauch der menschlichen Sprache. Einerseits handelt es sich im Gebrauch der Sprache immer um die Verknüpfung von Signifikanten (in Abbildung 2 durch S und S' symbolisiert) zu einer Kette - ein Prozess, der zeitlich einer diachronen Logik folgt (in Abbildung 2 durch den waagrechten Pfeil dargestellt). Andererseits wird Sprache nur dann erzeugt, wenn eine Person den Drang verspürt, eine Absicht oder ein Bedürfnis zu artikulieren (in Abbildung 2 das « $\Delta »$ an der Basis des wiederkehrenden Pfeils). Der wiederkehrende Pfeil in Abbildung 2 illustriert, wie diese Absicht mit der Zeit zur Erzeugung von Bedeutung und Subjektivität führt, und aus diesem Grunde sind die zwei Kreuzungspunkte zwischen den Pfeilen von entscheidender Bedeutung.

Der rechte Kreuzungspunkt (in Abbildung 2 als A bezeichnet) bezieht sich auf den Anderen ( Autre») oder den «Locus des Signifikantenschatzes» (Lacan 1960/1986, S.193) und macht deutlich, dass bei der Erzeugung von Sprache die Signifikanten dem uns zurVerfügung stehenden Lexikon entnommen werden. Der Sprechende verbindet diese Signifikanten zu einer Kette, und damit beginnt die Antizipation der Bedeutung. Der Gebrauch von Signifikanten lässt das Auftauchen einer Botschaft erwarten. Aber solange der bedeutungsstiftende Prozess noch im Entstehen begriffen ist oder herausgeschoben wird, wird auch die tatsächliche Bedeutung suspendiert (Lacan, 1966/1986, S. 64).

Der linke Kreuzungspunkt bezeichnet den Moment der Interpunktion, in welchem sich aus der Absicht zu sprechen mit der Zeit eine Bedeutung herauskristallisiert. Lacan hebt hervor, dass die Interpunktion, zeitlich gesehen, einer retroakiven Logik folgt. Bedeutung kann nur entstehen, wenn eine genügende Anzahl von Signifikanten artikuliert worden ist. Später artikulierte Signifikanten bestimmen demnach die endgültige Bedeutung der vorausgehenden. So antizi- 
Figure 2 Schema der Logik der Signifikation nach Lacan

(1998, 1960/2006c)

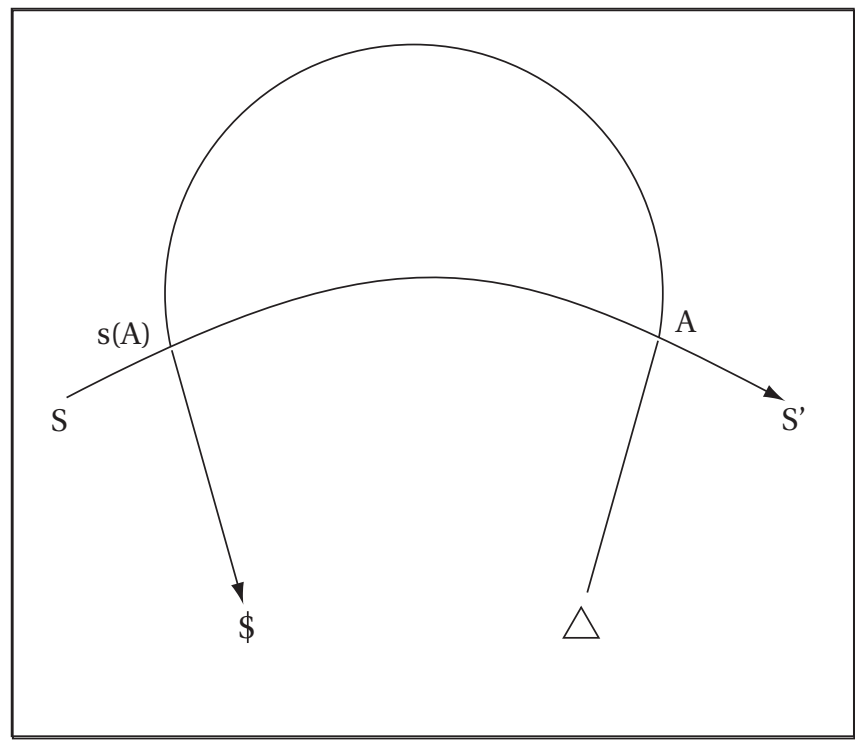

pieren wir beispielsweise im Satz «Du bist mein Freund... nicht mehr» anhand der ersten vier Signifikanten, dass es sich um eine Botschaft über Freundschaft handeln wird, aber die letzten beiden Worte verweisen auf das Gegenteil und bestimmen, dass im Endeffekteine Botschaft über Trennung übermittelt wird.

Schliesslich bewirkt der bedeutungsstiftende Prozess auch die Erzeugung von Subjektivität im Sinne Lacans. Die während des Prozesses artikulierten Signifikanten markieren und konnotieren zwar die implizierten Signifikanten, aber diese werden niemals genau gekennzeichnet. Lacan betrachtet das menschliche Subjekt als einen Effekt dieser Konnotation und schliesst daraus, dass das Subjekt gespalten ist (das durchgestrichene S in Figur 2). Das sich durch das Sprechen signifizierende Subjekt wird grundsätzlich über die Signifikanten zerstreut oder verteilt (Lacan 1960/1986). In der Neurose wird diese Spaltung typischerweise als intern erlebt, woraus sich die neurotische Tendenz zurVerdrängung erklären lässt; in der Psychose hingegen erscheint sie als von den eigenen Absichten losgelöst und von aussen kommend (Fink 1996; Nobus 2000; Vanheule 2011; Verhaeghe 2004). 


\section{Eine Halluzination ist ein aus der Kette gefallener Signifikant - Teil II}

Von seiner Theorie der Logik des Signifizierens ausgehend, gelangt Lacan zum präziseren Argument, dass das halluzinierte «perceptum» grundsätzlich «ein aus der Kette gefallener Signifikant» sei, und er schlägt vor, die Logik der halluzinatorischen Erfahrung in Bezug auf eine Unterbrechung in der Signifikantenkette zu verstehen (1966/1986, S. 65).

Der Fall der jungen paranoiden Frau, welche bei der Begegnung mit dem Liebhaber ihrer Nachbarin im Flur ihres Mietshauses den Begriff «Sau» halluziniert, illustriert demnach, worum es sich hier handelt. Da «Sau» ein aus der Kette gefallener Signifikant ist, rät uns Lacan, jede entsprechende Intervention als einen Versuch zu betrachten, diesen Signifikanten innerhalb der Signifikantenkette, aus der er hervorgegangen ist, zu verorten ${ }^{2}$. Folglich fragt er seine Patientin, was sie selbst an jenem Tag im Flur gesagt habe. Was die Halluzination betrifft, ist diese Art von Intervention diachronisch und bezweckt, die Halluzination genau in jenem Kontext zu situieren, welche zu ihrer Erzeugung geführt hat. "Nicht ohne Erfolg, denn sie gestand uns mit einem Lächeln, dass sie angesichts des Mannes tatsächlich jene Worte geflüstert hatte, die er, wenn man ihr glauben will, nicht so übel hätte aufnehmen dürfen: ‘Ich komme vom Metzger (...)» (Lacan 1959/1986, S. 66). Für Lacan ist der Satz "Ich komme vom Metzger» anspielungsreich und unvollständig. Anspielungsreich, weil die Patientin Mühe hat, die Bedeutung des Satzes zu präzisieren und zu begründen, warum sie ihn geäussert habe. Ferner betont er, dass der Satz auch grammatikalisch gesehen - und das ist von grundlegender Bedeutung - eine Anspielung enthält. Da es sich um ihre eigene Äusserung handelt, wird die Patientin mit dem Pronomen «Ich» als Subjekt des Satzes eingeführt. Aber das Prädikat «komme vom Metzger» vermag es nicht, ihr eine bedeutungsvolle Position gegenüber dem betreffenden Mann mit seinem zweifelhaften Ruf im Flur zuzuweisen. In diesem Satz schildert sie zwar, was sie an jenem Tag gemacht hat, beschreibt aber nicht adäquat, wer sie ist und was sie durchlebt. Darum kann er als unvollständig betrachtet werden. Durch die Begegnung im Flur wird sie als Frau in Bezug auf einen Mann eingeführt, dessen Art des Geniessens ihr fremd ist.

In Lacans Terminologie von 1959 kann der Mann als ein «Anderer» erkannt werden, insofern er jemand ist, den sie nicht verstehen oder in ihr Selbstbild einordnen kann. Kennzeichnend für eine solche Begegnung mit einem Anderen (an-Other) ist, dass dabei die eigene «Existenz als Subjekt» (1981/1997, S. 63) in Frage gestellt wird. Wer ist sie, als Frau, in Bezug auf Männer? Und allgemeiner: Was verbindet einen Mann und eine Frau? Doch genau in der Konfrontation mit der Frage nach ihrer Existenz als Subjekt wird die identitätsstiftende Interpunktion aufgeschoben. 
In Bezug auf die Logik des Signifizierens impliziert dies, dass der antizipatorische Aufschub, der dem Gebrauch des Signifikanten innewohnt, in der Schwebe bleibt. Lacan (1981/1993) weist darauf hin, dass dies typischerweise zu einem Gefühl von etwas Rätselhaftem sowie einer Spannung im psychotischen Subjekt führt. Der formulierte Satz signifiziert nicht adäquat die Absicht, welche die Frau veranlasste, sich an jenen Mann zu wenden und illustriert ihrVersagen, in Bezug auf den Mann, dem sie begegnet, eine begehrende Position einzunehmen ${ }^{3}$.

In Lacans späterer Formulierung kann dieser Mann als «Anderer des Geniessens» (Lacan 1966/2001, S. 215; Vanheule 2011) erkannt werden. Das heisst, dass seine Gegenwart die Patientin nicht nur in Frage stellt, sondern dass er in erster Linie eine libidinöse Aufladung verkörpert, die sie anhand der Signifikanten, mit welchen sie ihre eigene Existenz signifiziert, nicht begreifen kann. So gesehen ist die durch diese Situation entstehende Spannung nicht nur der Ausdruck einer misslungenen Signifikation. Sie zeugt von einer Konfrontation mit dem Drängen des Triebs, von welchem der Signifikant abbricht.

Lacan argumentiert, dass das Auftauchen des Signifikanten «Sau» im Realen diese Spannung auflöst und die Suspendierung des Konstituenten «Ich komme vom Metzger» retroaktiv bindet. Das plötzliche Auftauchen des halluzinierten Signifikanten setzt die bisher aufgeschobene Interpunktion ein und ist somit bedeutungsstiftend. Von nun an wird das Prädikat Sau die Identität des Ich signifizieren, welches zuerst als Subjekt des Satzes eingeführt wurde. Die damit empfangene Botschaft bedeutet eine Gleichstellung von «Ich» und «Sau»; und deshalb kommt die Frau zum Schluss, man habe sie beleidigt. Lacan (1986/1966) weist darauf hin, dass die Gewissheit über die schliesslich empfangene Botschaft proportional bis zu dem Zeitpunkt zunimmt, der als Abschluss des Prozesses der Interpunktion gelten kann.

Typisch für diesen Prozess des unterbrochenen Signifizierens ist, dass er von einer radikalen Unmöglichkeit zeugt, zu benennen, um was es geht. Lacan (1966/1986) erklärt, dass im Falle der Psychose ein fundmentaler Signifikant «verworfen» wurde. Das Konzept der «Verwerfung» muss demnach als das Gegenteil von Freuds «Bejahung» verstanden werden (Grigg 2008, 1981/ 1993; Maleval 2000; Verhaege 2004). Das Konzept der «Bejahung» drückt aus, dass ein Triebabkömmling in eine Repräsentation oder in einen Signifikanten transkribiert worden ist und folglich vom Subjekt als Element seines mentalen Lebens erkannt wird. Das Konzept der Verwerfung deutet darauf hin, dass eine solche Transkription nicht stattgefunden hat und dass bestimmte Elemente in Bezug auf das Subjekt vollkommen fremdartig, fremd, oder in Lacans Formulierung «real» bleiben. Oder/und noch 
genauer: Beim Signifikanten, der in der Psychose verworfen wird, handelt es sich um den «Namen-des-Vaters» ${ }^{4}$. Das Konzept des «Namen[s]-des-Vaters» bezieht sich auf das, was das Subjekt ins gemeinsame normative Universum, in dem wir leben, integriert (Grigg 2008; Maleval 2000; Vanheule 2011). Den Namen-des-Vaters anzunehmen impliziert, dass ein Subjekt sein Leben nach einer bestimmten Anzahl von Vorstellungen und Gesetzen richten kann, welche in einer bestimmten (Sub-) Kultur menschliche Interaktionen festlegen. Dies führt zu einer Aneignung oder Einverleibung von Vorstellungen über fundamental existenzielle Fragen, wie zum Beispiel die Bedeutung des Lebens angesichts des Todes, die Frage, wie Menschen zueinander in Beziehung stehen sollten, oder, wie Frauen und Männer eigentlich sind (siehe Lacan, 1959/1986). Die normativen Vorstellungen, die uns mit dem «Name[n]-des-Vaters» zur Verfügung gestellt werden, erlauben es, die mit diesen Fragen verbundene grundsätzliche Unsicherheit zu ertragen und ermöglichen dem Subjekt in einer nächsten Phase, als begehrendes Wesen seine eigenen Urteile und Entscheidungen zu fällen. Weil in der Psychose der «Name-des-Vaters» verworfen wird, fehlt dem psychotischen Subjekt nicht nur ein elementares Repertoire an Antworten auf diese existenziellen Themen, sondern - und dies ist noch fundamentaler - es hat eine andere Beziehung zum Symbolischen und zum Anderen, was sich in den Halluzinationen manifestiert.

Lacan (1959/1986) zeigt auf, dass eine Konfrontation mit Fragen, die auf die eigene Existenz als Subjekt hinweisen und mit der Erwartung einer persönlichen Positionierung einhergehen, die radikale Unfähigkeit des psychotischen Subjekts zu symbolisieren veranschaulicht und ein Gefühl der Ratlosigkeit hervorruft. Eine solche Konfrontation evoziert die Unmöglichkeit, die momentane Situation zu signifizieren und führt zu einer Unterbrechung im Prozess des Signifizierens; die Signifikantenkette benennt nicht mehr, worum es geht, und das Identitätsgefühl wird suspendiert. Diese Unterbrechung führt zum Erleben von Spannung und Rätselhaftigkeit, welches durch das Auftauchen eines aus der Kette gefallenen Signifikanten im Realen schliesslich benannt werden kann.

\section{Was Schreber uns lehrt: Botschaftsphänomene und Code-Phänomene} in Halluzinationen

Das autobiographische Buch von Daniel Paul Schreber (1903/2000), das auch von Freud (1911/1958) kommentiert wurde, ist für Lacan eine der wichtigsten Quellen; nicht nur, um diese Konzepte der Psychose im Allgemeinen auszuarbeiten, sondern besonders für die Erforschung der Halluzinationen. Lacan (1959/1986) greift auf Schrebers Autobiographie zurück, um die Struktur der 
verbalen Halluzinationen weiter zu untersuchen und konzentriert sich auf die Art und Weise, mit der Schreber seine Halluzinationen beschreibt. Ausgehend von seiner Theorie der Logik des Signifizierens unterscheidet Lacan (1959/1986) bei Schreber zwei typische Arten von Halluzinationen: Botschaftsphänome und Codephänomene.

Einerseits erkennt er bei Schreber Halluzinationen, die ähnliche Strukturen aufweisen wie unser oben beschriebener Fall der halluzinierenden Frau; Halluzinationen, bei welchen die aufgeschobene Interpunktion entscheidend ist. Diesbezüglich ist die Passage aus Schrebers Buch (1903/1973, S. 241) entscheidend, in welcher er schildert, wie unfertige Sätze und fragmentierte Ideen von aussen auf ihn einwirken, durch die «Strahlen», welche ihn mit Gott verbinden. So halluziniert er beispielsweise den Satzteil: «Nun will ich mich» und fühlt sich gezwungen, das Fragment mit einer Antwort wie «darein ergeben, dass ich dumm bin» zu vervollständigen. Lacan (1981/1997) betrachtet diese unfertigen Sätze als Zeugnis von Unterbrechungen im bedeutungsstiftenden Prozess und nennt sie Botschaftsphänomene. In einem Aspekt jedoch unterscheiden sich die unterbrochenen Botschaften, die Schreber in seinem Buch beschreibt, von den halluzinatorischen Erfahrungen der Frau aus Lacans Fallvorstellung. Während Schreber den ersten Teil des Satzes hört und ihn mit seinen eigenen zusätzlichen Signifikanten ergänzt, äussert die halluzinierende Frau die ersten Signifikanten und hört dann einen Zusatz. Auf der strukturalen Ebene verfügen beide Halluzinationen allerdings über eine Anzahl von gemeinsamen Eigenschaften. In beiden Fällen missglückt die Übermittlung einer Botschaft, obwohl die Antizipation von Bedeutung bereits in Gang ist. Und in beiden Fällen führt diese Unterbrechung beim Subjekt zu einem rätselhaften Zustand, zu einem Gefühl, in der Luft zu hängen. Lacan (1981/1997) schliesst daraus, dass, wie im Falle seiner Patientin, die Sätze an jener Stelle unterbrochen werden, in welcher das Subjekt zwar eingebracht, aber noch nicht durch eine Anzahl Attribute definiert ist. Der unvollendete Satz enthält eine «protasis», welche das Subjekt einführt, aber dessen Definition aufschiebt. Die «apodosis», welche dem Subjekt schliesslich Eigenschaften zuschreiben und damit den Satz vollenden könnte, fehlt jedes Mal. Daraus ergibt sich eine Störung in der Erzeugung einer Botschaft: Daher das Konzept der Botschaftsphänomene.

In seiner Diskussion des unvollständigen Satzes folgert Lacan zudem, dass Botschaften typischerweise dann abbrechen, wenn das Subjekt durch ein Personalpronomen oder, wie er es nennt, einen "shifter" eingeführt wird: «(...) dass der Satz an dem Punkt unterbrochen wird, wo die Wortgruppe endet, bei der man von Index-Termen sprechen könnte, d.h. von jenen Termen, die durch ihre Funktion

Psychosen - Psychoanalytische Perspektiven 
im Signifikanten - (...) als shifters ausgewiesen werden» (Lacan 1959/1986, S. 72). Genau genommen ist diese Aussage nicht korrekt. Schreber selbst (1903/2000. S 241.) berichtet von "protasen» wie "das war nu nämlich» und "fehlt uns nun», in welchen kein Personalpronomen auftaucht. (Diese Stellen werden von Lacan nicht zitiert). Von einem klinischen Gesichtspunkt aus gesehen ist die Vorstellung, dass Halluzinationen dieser Art immer das Subjekt via Personalpronomen einführen, nicht haltbar. Der Beweis dafür kann in Zeugnissen der psychotischen Patientin ohne weiteres gefunden werden. Allerdings führen die unvollständigen Sätze in jedem Fall das Subjekt im grammatikalischen Sinne des Wortes ein. Bezeichnend für den unvollständigen Satz ist, dass er das grammatikalische Subjekt benennt oder darauf anspielt, dessen Eigenschaften aber nicht weiter definiert. Was die Logik des Signifizierens betrifft, welche Lacan $(1986,1966)$ parallel zu seinem Werk über Psychosen vollständig ausgearbeitet hat (1981/1987), kann die Wirkung auf das Subjekt im psychoanalytischen Sinne des Begriffs als indirekt betrachtet werden. Wie ich in der Diskussion von Figur 2 gezeigt habe, erzeugt jedes Signifizieren Subjektivität, da die artikulierten Signifikanten den Sprechenden und den Hörenden zwangsläufig konnotieren. Wenn der Prozess des Signifizierens selbst unterbrochen wird, wie in den unvollständigen Sätzen, wird diese Konnotation aufgeschoben. Auf der Ebene der Botschaft führt dies zu einer Suspendierung, und es findet keine identitätsstiftende Interpunktion statt, mit dem Ergebnis, dass die Person, um deren Rede-Akt es sich handelt, in Bezug auf den Anderen essenziell undefiniert bleibt.

Schliesslich unterscheidet Lacan in den von Schreber beschriebenen Halluzinationen auch «Code Phänomene». Sie beziehen sich auf grundlegende Veränderungen in der Reihe von Signifikanten, über die ein Subjekt verfügt. Durch seine Halluzinationen eignet sich Schreber eine neue Gruppe von Signifikanten an, wie «Nerven-Kontakt» oder «Grundsprache» (Schreber 1903/1973, S. 75/77), welche keine konventionelle Bedeutung haben und die er seinem gebräuchlichen Lexikon beifügt. Lacan (1966/1986) weist darauf hin, dass «Code-Phänomene in Neologismen der Form nach (i.e. neue Wortzusammensetzungen, die aber den Regeln der Muttersprache des Patienten folgen) und der Verwendung nach» (S. 69/70) aufgeteilt werden. Damit bezieht er sich auf die Tatsache, dass durch Halluzinationen Wörter mit einer absolut privaten Bedeutung ausgedrückt werden können, Wörter, die ausserhalb des Kontextes der Rede des Psychotikers keine verständliche Bedeutung haben, die aber einen wesentlichen Aspekt seines Erlebens kennzeichnen. Neologismen benennen Aspekte der Erfahrung, welche durch die Verwerfung des «Namen[s]-des-Vaters» nicht auf eine herkömmliche Art benannt 
werden können. So benützt Schreber beispielsweise den Neologismus «NervenKontakt», um den aussergewöhnlichen und körperlich empfundenen Kontakt zu benennen, den er mit Gott zu haben glaubt.

Ich fasse zusammen: Die Unterscheidung zwischen Botschaftsphänomenen und Codephänomenen verdeutlicht, dass die aus der Kette gefallenen Signifikanten, die für die psychotischen Halluzinationen typisch sind, von zwei Prozessen zeugen können: von einer Störung bei der Interpunktion und einer grundlegenden Unfähigkeit, das eigene Leben und Sein zu signifizieren (Botschaftsphänomene), oder von privaten Begriffen, welche das eigene Leben und Sein sehr wohl zu signifizieren vermögen (Codephänomene).

\section{Schlussfolgerungen}

In diesem Aufsatz habe ich Lacans Theorie der Halluzinationen untersucht und sie klassischen psychiatrischen Ansätzen zu diesem Thema gegenübergestellt. Ich habe argumentiert, dass das psychiatrische Konzept der Halluzinationen gewöhnlich von phänomenologischen Definitionen ausgeht, welche die Unwirklichkeit der halluzinatorischen Wahrnehmungen hervorheben und Hand in Hand gehen mit einer anhaltenden Suche nach dem, was ihnen zugrunde liegt. Diese Suche taucht in frühen psychiatrischen Betrachtungen der Halluzinationen auf, setzt sich aber hartnäckig in der gegenwärtigen psychiatrischen Forschung fort. Lacan bricht mit dieser Tradition und untersucht die subjektive Wirkung der Halluzinationen auf die Person, die sie erleidet. Er betrachtet eine Halluzination als ein «perceptum» mit einer paradoxen Wirkung auf das «percipiens». Es handelt sich nicht um ein objektloses «perceptum», sondern um ein "perceptum», welches das Subjekt verstört. Lacan unterstreicht die Wirkungen der durch die Halluzinationen evozierten Perplexität und untersucht diese Wirkungen hinsichtlich einer unterbrochenen Signifikation.

Innerhalb dieser Logik betont Lacan, dass Halluzinationen von der Unmöglichkeit der psychotischen Personen zeugen, eine begehrende Position einzunehmen, wenn sie mit persönlich berührenden Situationen und triebverwandten Themen konfrontiert werden. Solche Konfrontationen offenbaren ein radikales Scheitern der Symbolisierung. Innerhalb dieser Logik gründen Halluzinationen in der Unfähigkeit, die eigene Existenz als ein Subjekt in Bezug auf ein anderes zu signifizieren. Die Signifikantenkette vermag nicht zu benennen, worum es sich bei einem Subjekt in der Konfrontation mit dem Anderen handelt. Diese Unterbrechung führt zu einer Suspendierung des Identitätsgefühls der psychotischen Person und zu einer Erfahrung von Spannung und Rätselhaftigkeit, dem das Auftauchen 
eines aus der Kette gefallenen oder halluzinierten Signifikanten schliesslich einen Namen verleiht. Dabei wurden zwei Arten von Halluzinationen unterschieden: Botschaftsphänomene und Codephänomene. Botschaftsphänomene sind das Ergebnis einer Störung bei der Interpunktion und einer grundlegenden Unfähigkeit, das eigene Leben und Sein zu signifizieren. Codephänomene bezeichnen eine höchst private Benennung, welche das eigene Leben und Sein durchaus zu signifizieren vermögen. Auf der Logik der unterbrochenen Signifikation aufbauend hat Lacan sich zu einem späteren Zeitpunkt auch mit der stimmartigen Eigenschaft der Halluzinationen befasst. Damit will er aufzeigen, dass es sich bei dem durch die Konfrontation mit triebverwandten Fragen evozierten Überschuss von Spannung oder «jouissance» genau um das handelt, was schliesslich in der Halluzination wiederkehrt (Vanheule 2011).

Innovativ in diesen Konzepten ist, dass die halluzinierende Person aus einer anderen Perspektive betrachtet wird, einer Perspektive, aus welcher Halluzinationen nicht automatisch auf den Status von Krankheitssymptomen reduziert werden, die durch einen desintegrierten Geist oder ein gestörtes Gehirn geschaffen werden. Lacan macht geltend, dass Halluzinationen von einer inneren Struktur zeugen, die genau widerspiegelt, womit sich die halluzinierende Person herumplagt. Er verzichtet auf jede semantische Interpretation und jeden Versuch des empathischen Verstehens, besteht aber auf genauem Hinhören: Halluzinationen sollen aufs Wort genau gelesen werden, und Kliniker sollen den Kontext, in dem sie entstanden sind, gründlich prüfen. Lacans Fokus liegt auf den Unterbrechungen im Prozess des Signifizierens, und er betont, dass diese Unterbrechung jeweils von Fall zu Fall untersucht werden soll. Psychoanalytiker dürfen nicht vor der Arbeit mit psychotischen Personen zurückschrecken; sie sollten ihren Erfahrungen zuhören, sie aber keinesfalls interpretieren. Stattdessen konzentriert sich die lacansche Behandlung psychotischer Patienten darauf, ihnen dabei zu helfen, eine Antwort auf das sie überwältigende Erleben zu finden (Apollon, Bergeron \& Cantin 2000; Maleval 2000; Vanheule 2011).

\section{Literatur}

Allen, P., Laroi, F., McGuire, P. K., \& Aleman, A. (2008). The hallucinating brain: A review of structural and functional neuroimaging studies of hallucinations. Neuroscience and Behavioral Reviews, 32, 175-191.

Apollon, W., Bergeron, D., \& Cantin, L. (2000). The treatment of psychosis. In K. R. Malone \& S. R. Friedlander (Eds.), The subject of Lacan - A Lacanian reader for psychologists (pp. 209-227). Albany: State University of New York Press. 
Atkinson, J. R. (2006). The perceptual characteristics of voice-hallucinations in deaf people: Insights into the nature of subvocal thought and sensory feedback loops. Schizophrenia Bulletin, 32, 701-708.

Berrios, G.E. (1996). The History of Mental Symptoms. Descriptive Psychopathology since the Nineteenth Century. Cambridge: Cambridge University Press.

David, A. S. (2004). The cognitive neuropsychiatry of auditory verbal hallucinations: An overview. Cognitive Neuropsychiatry, 9, 107-123.

Esquirol, E. (1838). Des maladies mentales considérées sous le rapport médical, hygiénique et médico-légal (Vol. 2) [On mental illnesses considered from a medical, hygienic and medical-legislative angle (Vol. 2)]. Paris, France: J.B. Baillère.

Ey, H. (1973). Traité des hallucinations (Tome 1) [Treatise on hallucinations (Vol. 1)]. Paris, France: Masson.

Fink, B. (1996). The Lacanian subject. Princeton, NJ: Princeton University Press.

Fink, B. (1997). A clinical introduction to Lacanian psychoanalysis: Theory and technique. Cambridge, MA: Harvard University Press.

Freud, S. (1958). Psycho-analytic notes on an autobiographical account of a case of paranoia. In J. Strachey (Ed. \& Trans.). The standard edition of the complete psychological works of Sigmund Freud (Vol. 12, pp. 9-82). London, England: Hogarth Press. (Original work published 1911)

Grigg, R. (2008). Lacan, language and philosophy. Albany: State University of New York Press.

Guiraud, P. (1950). Psychiatrie générale [General psychiatry]. Paris, France: Le François.

Kusnierek, M. (2008). Jacques Lacan's theory of perception. Bulletin of the NLS, 3, 22-35.

Lacan, J. (1993). The seminar 1955-1956: Book III. The psychoses (J. A. Miller, Ed. \& R. Grigg, Trans.). New York, NY: Norton. (Original work published 1981)

Lacan, J. (1998). Le séminaire 1957-1958: Livre V. Les formations de l'inconscient [The seminar 1957-1958: BookV. The formations of the unconscious]. Paris, France: Seuil.

Lacan, J. (2001). Présentation des mémoires d un névropathe [Presentation of the memoirs of a madman]. In Autres Écrits [Other écrits] (pp. 213-217). Paris, France: Seuil. (Original work published 1966)

Lacan, J. (2006a). On a question prior to any possible treatment of psychosis. In B. Fink (Trans.) Écrits (pp. 445-488). New York, NY: W. W. Norton. (Original work published 1959)

Psychosen - Psychoanalytische Perspektiven 
Lacan, J. (2006b). The mirror stage as formative of the I function as revealed in psychoanalytic practice. In B. Fink (Trans.) Écrits (pp. 75-81). New York, NY: W. W. Norton. (Original work published 1949)

Lacan, J. (2006c). The subversion of the subject and the dialectic of desire in the Freudian unconscious. In B. Fink (Trans.) Écrits (pp. 671-702). New York, NY: W. W. Norton. (Original work published 1960)

Maleval, J. C. (2000). La forclusion du Nom-du-Père [The forclusion of the the name-of-the-father]. Paris, France: Seuil.

Merleau-Ponty, M. (1962). The phenomenology of perception (C. Smith, Trans.). London, England: Routledge \& Kegan Paul. (Original work published 1945)

Miller, J. A. (1980). Teachings of the case presentation. In S. Schneiderman (Ed.) How Lacan's ideas are used in clinical practice (pp. 42-52). New Haven, CT: Yale University.

Miller, J. A. (2001). The logic of the perceived. Psychoanalytical Notebooks, 6, 9-30.

Nobus, D. (2000). Jacques Lacan and the Freudian practice of psychoanalysis. New York, NY: Routledge.

Schreber, D. P. (2000). Memoirs of my nervous illness. NewYork, NY: NewYork Review of Books. (Original work published 1903)

Stump, E. (2003). Aquinas. New York, NY: Routledge.

Vanheule, S. (2011). The Subject of Psychosis - A Lacanian Perspective. New York \& London: Palgrave Macmillan.

Verhaeghe, P. (2004). On being normal and other disorders. New York, NY: Other Press.

\section{Anmerkungen}

1 Artikel aus dem Englischen übersetzt von Patricia Kunstenaar.

2 Aufgrund seines Konzeptes einer fundamentalen Spaltung zwischen dem halluzinierten perceptum und dem percipiens spricht sich Lacan gegen jede psychologische Intervention aus, die auf eine Kontinuität zwischen beiden hinsteuert und damit den Fokus auf die Bedeutung des halluzinierten Signifikanten legt. So lehnt er beispielsweise eine Deutung des Wortes Sau gemäss dem freudschen Konzept der Projektion entschieden ab (die Patientin denkt eigentlich, der Liebhaber ihrer Nachbarin sei ein schmutziges Schwein, weil er verheiratet ist und trotzdem ganz offensichtlich ein Verhältnis mit ihr hat; diese Idee wird verworfen und kehrt von aussen als eine Botschaft, die ihm zugeschrieben wird, zurück) (Lacan, 1981/1997).

3 Entsprechend seinem Konzept (1981/1997), dass die Struktur des Symptoms einer Person tatsächlich die Struktur ihrer Pathologie widerspiegelt, sieht Lacan die Unfähigkeit seiner Patientin, als Frau einem Mann gegenüber eine begehrende Position einzunehmen, als Quelle ihrer paranoiden Gewissheit. Sie war erst vor kurzem wieder zu ihrer Mutter gezogen, 
besessen von der Überzeugung, ihr Mann und seine Familie hätten die Absicht, sie in Stücke zu zerschneiden - wie die Bauern auf dem Lande es mit den Schweinen zu tun pflegten.

4 Lacan betrachtet den Ödipus-Komplex als Metapher, in welcher der Name-des-Vaters ein zentraler Signifikant ist, der einen anderen Signifikanten ersetzt - das Begehren der Mutter, welches bis anhin für das Kind rätselhaft war. Er wird als fundamentaler Signifikant dargestellt, der ein Modell des Benennens durch metaphorische Substitution einführt und dem Prozess des Signifizierens einen normalen Verlauf ermöglicht. Der Name-des-Vaters ist zugleich auch der Signifikant, welcher die Gesetze und Verbote verkörpert, die unsere Beziehungen $\mathrm{zu}$ andern strukturieren. Er ordnet das Subjekt in die symbolische Ordnung der Realität ein. 